\title{
LA-UR-96- 3119
}

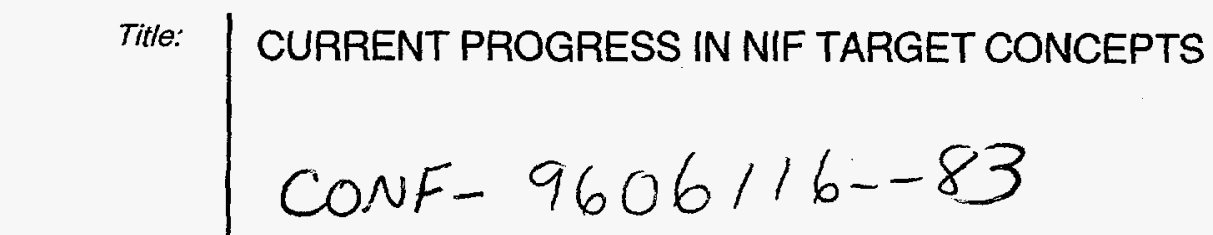

RECEIVED

SEP 231996

OSTI
Author(s):

Submitted to:

\section{Peter L. Gobby \\ MST-7 \\ Larry R. Foreman \\ Dan J. Thoma \\ MST-7 \\ MST-6 \\ Loren A. Jacobson MST-6 \\ Rebecca V. Hollis MST-7 \\ Michael A. Mitchell MST-7 \\ Mike A. Salazar MST-7 \\ Leander J. Salzer MST-7}

12th Topical Meeting on Fusion Energy of ANS

June $16-20$

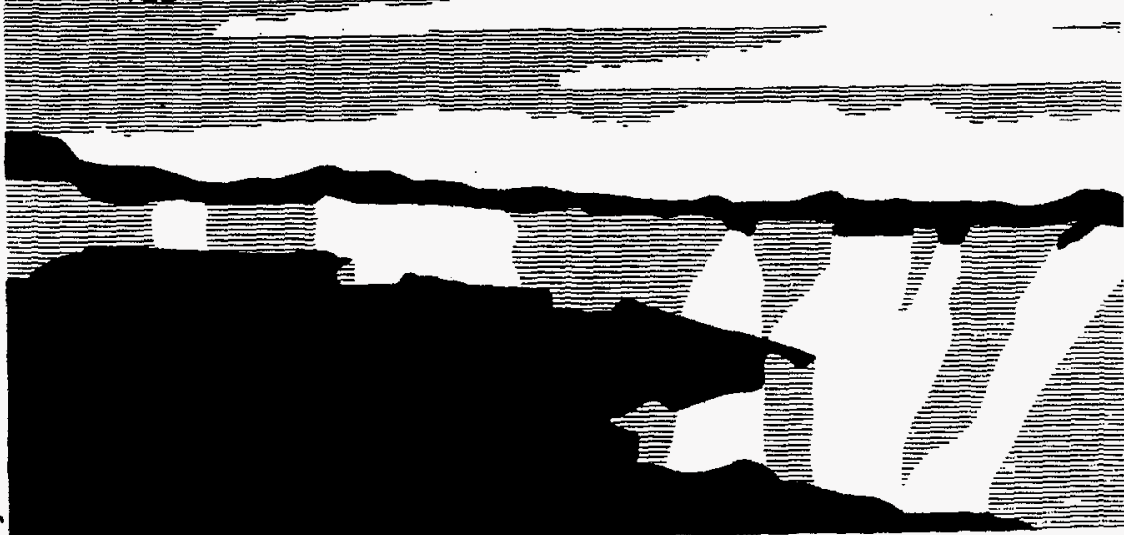

NATIONAL LABORATOR

Los Alamos National Laboratory, an affirmative action/equal opportunity employer, is operated by the University of California for the U.S. Department of Energy under contract W-7405-ENG-36. By acceptance of this article, the publisher recognizes that the U.S. Government retains a nonexclusive, royalty-free license to publish or reproduce the published form of this contribution, or to allow others to do so, for U.S. Government purposes. The Los Alamos National Laboratory requests that the publisher identify this article as work performed under the auspices of the U.S. Department of Energy. 


\section{DISCLAMER}

Portions of this document may be illegible in electronic image products. Images are produced from the best available original document. 


\title{
CURRENT PROGRESS IN NIF TARGET CONCEPTS
}

\author{
P.L. Gobby, L.R. Foreman, D.J. Thoma, L.A. Jacobson, R.V. Hollis, J. Barrera, \\ M.A. Mitchell, M.A. Salazar and L.J. Salzer \\ Materials Science and Technology Division \\ Los Alamos National Laboratory \\ Los Alamos, NM 87545
}

\begin{abstract}
Target concepts for the National Ignition Facility (NIF) require progress in the art and science of target fabrication. Three distinct issues are addressed in the work being described in this paper. They are (1) beryllium fuel capsules, (2) foam-buffered direct drive, and (3) high density gas-filled hohlraums. In all cases experiments on the existing Nova laser at Lawrence Livermore National Laboratory are either in progress or planned for the near future to test the various concepts. Consequently target fabrication must be able to deliver targets appropriate for each.
\end{abstract}

\section{Introduction}

A major emphasis of ICF target fabrication is to provide ignition targets for the proposed National Ignition Facility (NIF). Since the point design for such targets involves a highly uniform and smooth DT ice or liquid layer inside a low-z shell, the efforts to produce and characterize such DT layers has dominated the efforts. But there are other issues relevant to targets which are planned for the NIF. This paper discusses three such target concepts being pursued at Los Alamos: (1) beryllium fuel capsules, (2) foam-buffered direct drive, and (3) high density gas-filled hohlraums.

Beryllium capsules are interesting for a number of reasons, having some advantages over the baseline plastic capsules. First, at room temperature the DT pressure in a NIF ignition capsule is 300 atmospheres. Beryllium is inherently strong enough to hold this DT load at room temperature, while plastic capsules cannot. Second, the beta-layering process ${ }^{1}$ will result in the DT ice layer following the isotherms presented by the capsule. If the capsule material had infinite thermal conductivity, or a perfectly spherically symmetric cooling environment could be presented to the capsule, we could expect the isotherms in the capsule to be spherically symmetric as well. But the cylindrical hohlraum enclosing the capsule will surely present a somewhat asymmetric cooling environment, and all capsule materials under consideration have finite conductivities. Thus a high conductivity capsule will be preferable. At $20 \mathrm{~K}_{\text {pure beryllium }}{ }^{2}$ has a much higher thermal conductivity that any plastic ${ }^{3}$. If a beryllium alloy is used, its conductivity will be much poorer, but it is not unreasonable to expect that it will still surpass plastics. If the thermal conductivity of the beryllium alloy or the plastic is not sufficient to overcome the asymmetries of the cooling environment, then some form of "thermal shimming" will have to be added to the cooling environment to yield the desired spherical isotherms and the uniform DT layer.

Foam-buffered targets are being studied primarily to aid in direct drive fusion. Experiments indicate that a low density foam layer on the outer surface of the fuel capsule helps to ameliorate the effects of nonuniformities in the laser drive. ${ }^{4}$ We have fielded targets in 1,2 and 3-dimensional configurations to test this concept., and foams of polystyrene-divinylbenzene and also various acrylate foams have been used. The single common requirement is that the density be approximately $50 \mathrm{mg} / \mathrm{cc}$ and the cell size be $1-2$ microns.

Current NIF ignition designs use a heliumhydrogen mixture at a density of about 750 micrograms $/ \mathrm{cc}(5.5 \mathrm{~atm}$ at $300 \mathrm{~K})$ in the hohlraum surrounding the capsule. The main purpose of this gas is to tamp the wall motion that occurs as the hohlraum is being heated by the laser beams. It will also serve as a heat transfer medium in the cooling of the capsule. This gas presents other complications resulting in the potential reflection of laser energy out of the hohiraum. Numerous experiments continue to be fielded to study these phenomena. 5,6 Experiments to date have used higher molecular weight gases at low pressure (and ambient temperature) instead of high density $\mathrm{He} / \mathrm{H}_{2}$. 
Los Alamos researchers wish to test the actual $\mathrm{He} / \mathrm{H}_{2}$ mixtures at up to 3 times the $0.75 \mathrm{mg} / \mathrm{cc}$ density. This corresponds to $1 / 10 n_{C}$, where $n_{\mathcal{C}}$ is the critical electron density corresponding to the plasma frequency for the $3 \omega$ laser. This is desired because the expanding plasma from the NIF hohlraum wall will compress the gas to well above its starting level. Windows which are strong enough to withstand the 16-17 atm. require too much laser energy to burn through, so we are preparing a system to cool hohlraums to $<20 \mathrm{~K}$ to achieve the desired density at nearly 1 atmosphere. These experiments are planned for the Nova laser which was not designed to accommodate cryogenic targets. A relatively simple system which can be retrofitted onto the Nova target chamber is being fabricated. Cooled hohlraums filled with helium-hydrogen mixtures will attain the required density with pressures near 1 atmosphere. This allows relatively thin laser-entrance windows with minimal curvature.

\section{Beryllium Capsules}

Beryllium capsules calculationally demonstrate an advantage over plastic capsules. A number of issues must be addressed if the theoretical advantages of beryllium are to be realized. These include fabricability, surface finish, opacity control, joining and gas filling. All of these are being addressed at Los Alamos.

Two fabrication methods are currently being pursued at Los Alamos - direct machining of wrought beryllium and organometallic chemical vapor deposition (OMCVD). Figure 1 shows a pair of beryllium hemishells of NIF size ( $2 \mathrm{~mm}$ O.D., $0.1 \mathrm{~mm}$ wall) directly machined from wrought beryllium. A reversible, magnetically-loaded, kinematic mount ${ }^{7}$ attached to the lathe spindle allowed the machining and lapping of both the inner and outer surfaces of the hemishells. Initial measurements of this novel kinematic mount show that positioning reproducibility is within 0.2 micron. By using the highest precision machines and tooling to fabricate the parts of the kinematic mount, we believe that producing hemishells with concentricities of 0.25 micron or better is possible. The surface finish of the both inner and outer surfaces of shells produced thus far have been $<10 \mathrm{~nm} \mathrm{rms}$, as determined using a 3-d optical interferometer. The interferometer samples an area of about $10,000 \mu \mathrm{m}^{2}$, so a single such measurement does not yield the low mode variations of the surfaces. Measurements of these lower modes are planned for the future.
The OMCVD method is motivated partially by our experience in fluidized bed coating techniques, which yield highly uniform, high density coatings. The system under development uses dicyclopentadienylberyllium, $\mathrm{C}_{10} \mathrm{H}_{10} \mathrm{Be}$. Though not commercially available, it has been synthesized and the chemistry is straightforward. $8,9,10$ The most common of the dicyclopentadienyls is that of iron, commonly known as ferrocene. Preliminary experiments are being conducted on the magnesium analog, which has a similar chemistry to that of beryllium, but not its health hazards. The reduction of the organometallic will occur in a reducing hydrogen atmosphere at about $200 \mathrm{C}$. Our experience in CVD processes indicates that minimization of carbon and other organic in the deposits will be a major emphasis of this development. Since the fluidized bed technique is ideal for fully coating spherical mandrels, the removal of the mandrels will also demand significant effort.

The low atomic number of beryllium makes it a highly desirable ablation material, but if the beryllium is to ablate, it must first absorb the hohlraum radiation, and here the low $\mathrm{z}$ is a shortcoming. So beryllium doped with a small quantity of higher $z$ material is desirable. From a metallurgical viewpoint, copper is a good choice. Copper has the highest solubility of other metals in beryllium. ${ }^{11}$ The quoted solubilities are, however, for equilibrium conditions, and higher metastable solubilities are possible. Experiments are underway to determine the practical limit to copper solubility in beryllium.

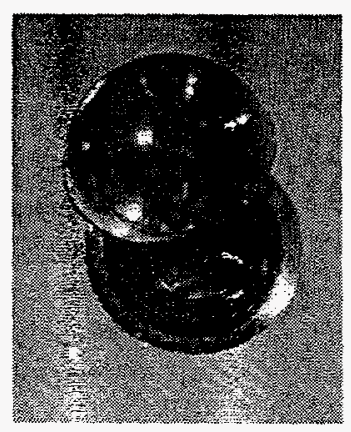

Fig. 1. Directly machined and polished beryllium hemishells. The outside diameter is $2.0 \mathrm{~mm}$.

Fabrication of the beryllium capsule (with the requisite high-z dopant) still leave the questions of joining and filling. The hemishells of Fig. 1 must be joined either by a weld, organic adhesive, braze or diffusion bond. We have had some success bonding beryllium with a thin aluminum braze. This requires the cleanliness of ultra-high vacuum and temperatures of $800 \mathrm{C}$, but acceptable bonds have been produced with as little as 3 
microns of aluminum. Diffusion bonding is also an option for beryllium. Our preliminary tests are encouraging. Welding or adhesive bonding have thus far not been considered.

The filling of a beryllium capsule with DT provides a challenge. The permeability of beryllium to hydrogen isotopes is negligible. So the permeation filling used with the plastic capsules is not a viable option. The joining of parts in a high pressure DT atmosphere greatly complicates the process and attempts to do this have been unsuccessful. Gluing a small beryllium plug into the capsules under the DT pressure can be done, but the minimum plug size we can fabricate may still be larger than designers can tolerate.

Another novel possibility involves a small palladium plug diffusion bonded into the beryllium. Palladium, which is known for its permeability to hydrogen, also alloys with beryllium making the diffusion bond possible. This would completely separate the fabrication and filling processes. This way a complete, characterized beryllium capsule with a palladium plug could later be diffusion filled with the DT. Filling a NIF capsule to 300 atmospheres would be slow through a 10 micron diameter palladium plug; extrapolation of data at lower pressures ${ }^{12}$ suggests a filling time on the order of one month. The density mis-match of the palladium, however, would require that the $\mathrm{Pd}$ pin would have to be very small (perhaps 5-10 microns diameter), so machining the pin and drilling the mating hole in the Be will not be trivial.

\section{Foam-buffered Targets}

If a foam layer is to help in smoothing the nonuniformities in direct drive laser beams, it must do so with a minimal energy penalty. Thus there cannot be a large mass represented by the foam (relative to the capsule). Also, it is generally accepted that the foam layer thickness must be the same order as the lateral dimensions of the non-uniformities if smoothing is to occur. Consequently, for targets fielded thus far, the foam layer thickness has varied from $50-200$ microns and the density from 10 $50 \mathrm{mg} / \mathrm{cc}$. In all cases the cell size is desired to be small (1-3 microns).

We have used two different foam systems to produce the targets required for these experiments polystyrene for spherical targets and acrylate foams for planar and cylindrical targets. This difference in fabrication techniques is driven by expedience - the acrylate foams are cast to size from a liquid precursor, which was easily applied to planar and cylindrical targets. The planar targets have all been fabricated with a washer bonded to a flat film. The opening in the washer was then filled with the precursor and cured. The cylindrical targets were parylene spools, produced by parylene coating aluminum mandrels. The surface tension of the foam precursor serves to counteract the effects of gravity and yield a uniform foam thickness. Under- or overfilling the spool results in a convex or concave, though azimuthally uniform, foam surface.

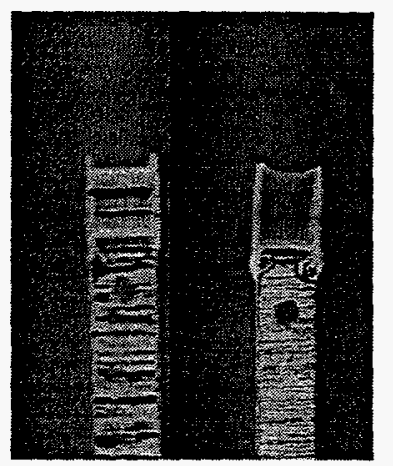

Fig. 2. Parylene spool (left) and foam-buffered parylene spool target. The concavity in the foam layer is due to an incomplete filling with the foam precursor. The spool i.d. is 350 microns.

The foam layers for the spherical targets were machined directly from larger pieces of polystyrene foam. Hemishells of the polystyrene foam were machined to 200 micron thickness with an inside diameter of approximately $1.5 \mathrm{~mm}$. (Fig. 3) These foam layers were then assembled directly over glass micro balloons (GMB's) containing 20 atmospheres of DT.

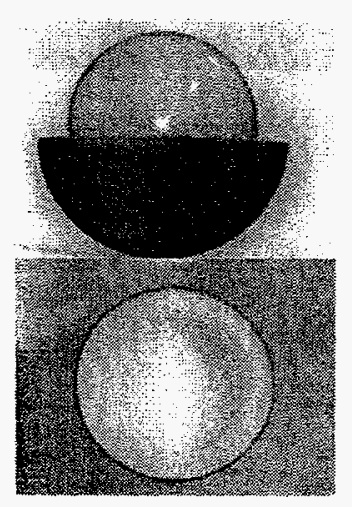

Fig. 3. Foam hemishells. The upper photo has the 1.6 $\mathrm{mm}$ GMB in place. The exteriors of the foam shells have been coated with 250 Angstroms of gold. 
The acrylate foam system was not used for these targets because the GMB's would not survive the 80-90 atmosphere external $\mathrm{CO}_{2}$ pressure that they would be subjected to during the supercritical extraction and drying step necessary for those foams.

Other foam processes are currently being considered to replace the machined polystyrene hemishells. Determination of the precise I.D. of the foam hemishells is difficult (the cell size is 1-3 microns), and it is difficult to prove the foam is in intimate contact with the GMB. This is an appeal of the acrylate system, where the casting in place and the negligible shrinkage boosts one's confidence that there is intimate contact between the $\mathrm{GMB}$ and the foam. We are currently considering casting the polystyrene around the GMB using a mold. Though more shrinkage is common to this system, the small size may yield intimate foam layers that do not crack or separate. Also the polystyrene foam is not subjected to the high external of supercritical drying, so our objection to using the acrylates is avoided.

\section{High Density Gas-filled Hohlraums}

We are currently developing a system to field high density gas-filled hohlraums at Nova. Cooling only the target will provide the high density required. A schematic of this simple system is shown in Fig. 4. This system will allow filling of the target to the approximately 1 atmosphere pressure before insertion into the Nova target chamber. This simplifies the fielding significantly, since no remotely actuated vaives are necessary, and the room temperature filled target is under no stress until it enters the target chamber vacuum. Then we need only monitor the pressure of the system and the temperature of the target, which starts at $300 \mathrm{~K}$ and is cooled to the 15 $20 \mathrm{~K}$ range. Initially the target, which has a volume of about $12.5 \mathrm{~mm}^{3}$, and the reservoir, with a volume of about $5000 \mathrm{~mm}^{3}$, are both a room temperature. There is no valve between the two, only a valve at the reservoir to accommodate filling. As the cryostat is cooled with liquid $\mathrm{He}$, only the target and its shield are cooled; the reservoir remains at room temperature. Because of the large reservoir-to-target volume ratio, cooling the target to low temperature increases the gas density in the target without any appreciable change in the system pressure. In fact, assuming ideal gases, the desired density of about $2.25 \mathrm{mg} / \mathrm{cc}$ for $\mathrm{He}-\mathrm{H}_{2}$ mixtures will occur at $15.5 \mathrm{~K}$ and $0.95 \mathrm{~atm}$.

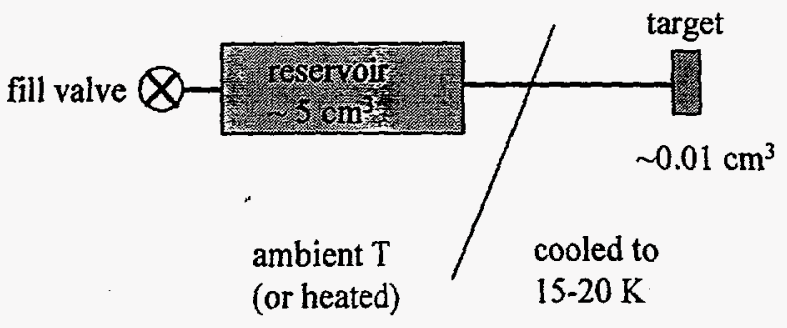

Fig. 4. Schematic diagram of the high density gas target system for Nova experiments.

But hydrogen, in particular, is not an ideal gas at these temperatures, and at a given partial pressure will begin to condense somewhere below $21 \mathrm{~K}$. Fig. 5 illustrates this point. Depending on the relative amount. of $\mathrm{H}_{2}$, the curve shows the temperature at which hydrogen would begin to condense (assuming the total gas density yields $1 / 10$ critical). To avoid this we need to stay above the curve. For example at $50-50 \mathrm{He} / \mathrm{H}_{2}$, if we wish to have $1 / 10$ critical density, we must keep the temperature no lower than $18.6 \mathrm{~K}$, at which point the total system pressure would be $1.15 \mathrm{~atm}$. To achieve this pressure we would either initially fill the system to $1.19 \mathrm{~atm}$ and cool the target to the $18.6 \mathrm{~K}$, or if we filled the system initially to $1 \mathrm{~atm}$, we'd heat the reservoir to $360 \mathrm{~K}$ while reducing the target temperature to $18.6 \mathrm{~K}$. Slightly higher pressures and temperatures can be used to achieve the $1 / 10$ critical density while safely avoiding hydrogen condensation.

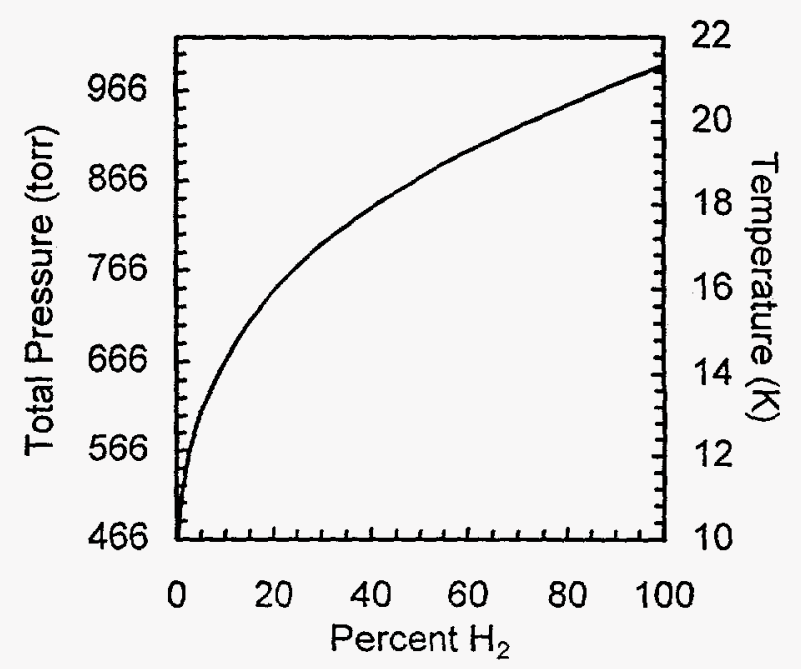

Fig. 5. Graph of hydrogen condensation temperatures for various $0.1 \mathrm{n}_{\mathrm{c}} \mathrm{He} / \mathrm{H}_{2}$ mixtures. To avoid hydrogen condensation the system must be operated above the line. 


\section{References}

1 J.K. Hoffer and L.R. Foreman, J. Vac. Sci. Technol. A 7 (1989) 1161.

2 A.I.P. Physics Handbook, 2nd ed.(1963) McGraw-Hill, New York, 4-90.

3 Chemical Engineer's Handbook, 6th ed.(1984) McGraw-Hill, New York, 3-261.

4 M. Dunne et al., Phys. Rev. Lett., 75 (1995)3858.

5 R.G. Watt et al., Phys. Plasma 3 (1996)1091.

6 H.A. Rose et al., Phys. Rev. Lett. 72 (1994)2883.

7 Patent applied for.

8 T.C. Bartke, U. of Wyoming Ph. D. Thesis (1975).

9 G.E. Parris and E.C. Ashby, J. of Organomettalic Chem. 72 (1994) 1.

${ }^{10}$ E.C. Ashby and R.C. Arnott, J. of Organomettalic Chem. 14 (1968) 1.

11 ASM Handbook, Alloy Phase

Diagrams, Vol. 3, (1992) ASM International, Materials Park, Ohio, pp.93-97.

12 Dushman, S., "Scientific Foundations of Vacuum Technique"(1958) John Wiley \& Sons, New York, pp 607-618. 